\title{
A network selection algorithm based on enhanced access router discovery in heterogeneous wireless networks
}

\begin{abstract}
The management process between different wireless technologies for mobile devices is very important to complete the handover operations. The handover operation needs to determine the delay and packet loss in order to be the quality of service within a certain level. Selecting the best available network at the appropriate time is very significant in the direction of realizing ubiquitous networks. In this paper a network selection approach named enhanced access router discovery (EARD) is proposed. The approach is developed to work in a heterogeneous environment including of WiMAX and WLAN networks. The EARD method utilizes the prioritized rating for multiple criteria (PRMC) proposed for selecting the target network. The proposed approach is evaluated with respect to various conditions with different traffic types. The simulation results show that our proposed approach outperform the traditional network selection methods is selecting the most appropriate network.
\end{abstract}

Keyword: Vertical handover; Heterogeneous wireless network; Network selection; Handover decision; Traffic class; EARD 